\title{
Clocks do not tick in unison: isolation of Clock and vrille shed new light on the clockwork model of the sand fly Lutzomyia longipalpis
}

João Silveira Moledo Gesto ${ }^{1,2}$, Gustavo Bueno da Silva Rivas ${ }^{1}$, Marcio Galvão Pavan ${ }^{1,3}$, Antonio Carlos Alves Meireles-Filho ${ }^{4}$, Paulo Roberto de Amoretty ${ }^{1}$, Nataly Araújo de Souza ${ }^{5}$, Rafaela Vieira Bruno ${ }^{1,6^{*}+}$ and Alexandre Afranio Peixoto ${ }^{1,6+}$

\begin{abstract}
Background: Behavior rhythms of insect vectors directly interfere with the dynamics of pathogen transmission to humans. The sand fly Lutzomyia longipalpis is the main vector of visceral leishmaniasis in America and concentrates its activity around dusk. Despite the accumulation of behavioral data, very little is known about the molecular bases of the clock mechanism in this species. This study aims to characterize, within an evolutionary perspective, two important circadian clock genes, Clock and vrille.

Findings: We have cloned and isolated the coding sequence of L. longipalpis' genes Clock and vrille. The former is structured in eight exons and encodes a protein of 696 amino acids, and the latter comprises three exons and translates to a protein of 469 amino acids. When compared to other insects' orthologues, L. longipalpis CLOCK shows a high degree of conservation in the functional domains bHLH and PAS, but a much shorter glutamine-rich (poly-Q) C-terminal region. As for L. longipalpis VRILLE, a high degree of conservation was found in the bZIP domain. To support these observations and provide an elegant view of the evolution of both genes in insects, phylogenetic analyses based on maximum-likelihood and Bayesian inferences were performed, corroborating the previously known insect systematics.

Conclusions: The isolation and phylogenetic analyses of Clock and vrille orthologues in L. longipalpis bring novel and important data to characterize this species' circadian clock. Interestingly, the poly-Q shortening observed in CLOCK suggests that its transcription activity might be impaired and we speculate if this effect could be compensated by other clock factors such as CYCLE.
\end{abstract}

Keywords: Lutzomyia longipalpis, Circadian clocks, Clock, vrille, PAS domain, bZIP domain, bHLH domain

\section{Findings}

Understanding the dynamics of pathogen transmission to the human hosts is a crucial step towards the development of effective tools to control vector-borne diseases. From this perspective, the study of vector behavior, as well as its genetics underpinnings, is key for any vector control program. One of the most relevant behavior traits across insect taxa is the circadian

\footnotetext{
* Correspondence: rafaelav@ioc.fiocruz.br

${ }^{\dagger}$ Equal contributors

'Laboratório de Biologia Molecular, Instituto Oswaldo Cruz, Fundação Oswaldo

Cruz (FIOCRUZ), Rio de Janeiro, Brazil

${ }^{6}$ Instituto Nacional de Ciência e Tecnologia em Entomologia Molecular

(INCT-EM)/CNPq, Rio de Janeiro, Brazil

Full list of author information is available at the end of the article
}

rhythmicity, which is depicted by well-defined daily patterns of locomotion, flight, adult emergence, olfactory response and other biological phenomena [1]. In tropical disease vectors, such as mosquitoes and sand flies, feeding habits and hematophagic behavior also follow a precise circadian schedule, often resulting in the outline of species-specific temporal niches [2-6].

For the last couple of decades, great effort has been made to unravel the molecular and cellular bases of the endogenous clock behind the circadian rhythms. In insects, the most comprehensive information regarding the molecular clock comes from the research with Drosophila melanogaster. Due to state-of-the-art genetic tools developed for this species, the core clock factors could be identified and fit in 
a pacemaker model consisting of self-sustained feedback loops that act on the regulation of gene expression $[7,8]$. Briefly, the transcription factors Clock ( $\mathrm{Clk})$ and cycle (cyc) interact at the pacemaker's core and activate the expression of several clock genes, such as period (per), timeless (tim), vrille (vri), Par domain protein 1 (Pdp1) and clockwork orange (cwo). Along the day, the proteins encoded by these genes feedback to modulate CLK/CYC transcription activity, thereby generating a molecular oscillation in their own expression. This model acquires extra layers of complexity with post-transcriptional and post-translational regulation, as well as cellular communication within neuronal clusters, providing a daily harmonious modulation of rhythmic behavior $[7,8]$.

Despite the good amount of data on the behavioral rhythms of tropical disease vectors [2-6], few studies have focused on the cellular and molecular mechanisms underlying them. The sand fly Lutzomyia longipalpis (Diptera, Psychodidae, Phlebotominae), main vector of visceral leishmaniasis in the New World, was the first insect vector to have clock components identified at the molecular level, with full characterization of the $c y c$ orthologue $[9,10]$ Interestingly, gene expression assessment of these components revealed aspects of the pacemaker that are different from the Drosophila model: 1) Clk mRNA peaks at an opposite phase in the two species; and 2) cycle expression exhibits a significant circadian oscillation in sand flies, whereas in Drosophila it is constitutive $[9,10]$. Nevertheless, these are probably not the only differences driving the distinct activity pattern between the two species, with $D$. melanogaster being mainly diurnal and L. longipalpis predominantly active at dusk/night [9]. The identification and functional characterization of other clock genes is key for the construction of a complete profile of the molecular pacemaker regulating the behavior in sand flies.

In the current work we report the isolation of the coding sequences of $\mathrm{Clk}$ and vri orthologues in L. longipalpis and their phylogenetic placement in Insecta (Neoptera infraclass) trees. Sand fly specimens were derived from a natural population of Lapinha Cave (longitude $43^{\circ} 57^{\prime} \mathrm{W}$, latitude $19^{\circ} 03^{\prime} \mathrm{S}$; approximate altitude $700 \mathrm{~m}$ ), a non-endemic area located in Sumidouro State Park, Minas Gerais, Brazil. Sand flies from this location were used as reference because they have been extensively studied and can be collected in greater abundance than other populations from endemic areas. Nucleotide sequences were amplified by PCR using both degenerate and specific primers (Additional file 1), following a "gene walking approach" according to Gentile et al. [11]. The 3' region was tackled by using the oligo-dT primer and the $5^{\prime}$ ' region by means of the " 5 ' Race System for Rapid Amplification of cDNA Ends" kit (Life Technologies), although the latter did not work well for Clk. In this case, the 5' region was obtained by PCR with the degenerate primer 5CLKdeg13, which anneals to the beginning of the coding sequence. Fragment sequences were subjected to fluorescent dye-terminator cycle sequencing reactions (ABI Prism ${ }^{\circ}$ BigDye ${ }^{\circ}$ Terminator v3.1 Cycle Sequencing Kit, Applied Biosystems), and run on an ABI 3730 automated sequencer. Nucleotide sequences were compared to the D. melanogaster database (www.flybase.org/blast), confirming homology to Clk and vri. Subsequently, these sequences were trimmed for poor quality fragments and plasmid sequences, and then assembled in unique contigs using the Staden software [12].

We have obtained the entire nucleotide coding sequence of L. longipalpis Clk and vri [GenBank: KR706373; KR706372]. L. longipalpis Clk encodes a putative protein sequence of 696 amino acids, enclosing the expected bHLH (basic Helix-Loop-Helix) (residues 12-63) and PAS (perarnt-sim) (residues 84-351) domains, the former involved in DNA binding and the latter in protein dimerization [13]. The glutamine-rich (poly-Q) region, which is believed to be involved in CLK transcription activation [13], is much shorter or even absent in this species. With respect to $L$. longipalpis' vri, the predicted protein sequence contains 469 amino acids and carries the characteristic bZIP (basic Leucine Zipper) domain (residues 112-168), which mediates DNA binding [14]. All these functional domains were confirmed using the Pfam v27.0 database [15].

Soon after the experimental isolation of $C l k$ and $v r i$ was achieved, the genome project of L. longipalpis released its first assembly (LlonJ1), including a number of draft contigs and scaffolds (https://www.vectorbase.org/organisms/ lutzomyia-longipalpis/jacobina/llonj1). Using our sequences as queries in BLAST searches, we were able to locate $C l k$ and vri in the LlonJ1.1 gene set. Due to misassembly issues, $\mathrm{Clk}$ sequence maps to two predicted genes: LLOTMP006614 (Scaffold48: 204225-210403 forward strand) and LLOTMP000502 (Scaffold108: 104,185106,138 forward strand), both incorrectly annotated. On the other hand, this was not an issue with vri, which is annotated as LLOTMP007416 (Scaffold568: 62113-76422 forward strand). The comparison of our sequences to the currently available genomic DNA allowed the identification of exons, introns and their boundaries. Hence, while $C l k$ is structured in 8 coding exons and 7 introns, vri contains 3 coding exons and 2 introns (Fig. 1).

In order to evaluate the degree of CLK and VRI conservation in insects, giving emphasis to vector species, the predicted protein sequences of L. longipalpis were aligned to their orthologous counterparts in eight neopteran insect species (Additional file 2), using the Multalin algorithm [16]. The Blosum62 substitution model [17], with default parameters of gap penalties imposed, was used to construct alignment matrices. No penalty was charged for terminal gaps. 


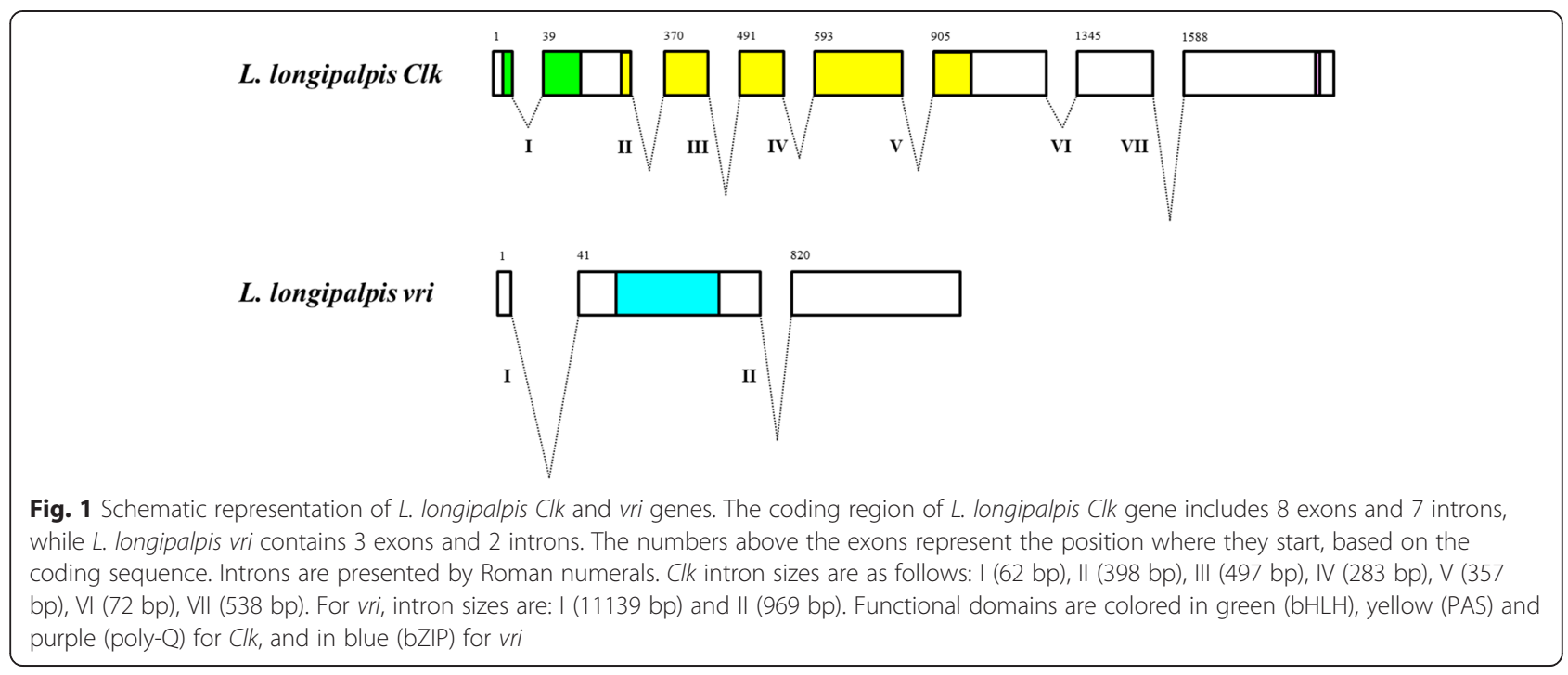

The multiple sequence alignment (MSA) of CLK orthologues (Additional file 3) revealed highly conserved regions related to bHLH and PAS functional domains, reaching an identity degree of 63 and $39 \%$, respectively, among the species analyzed. The C-Terminal region, on the other hand, is quite divergent, with low amino acid conservation. Equally variable is the size and structure of the poly- $\mathrm{Q}$ sequence found in this region, which is abundant in some species (e.g. D. melanogaster) but seems to be reduced or absent in L. longipalpis. The MSA of VRI orthologues (Additional file 4) also revealed regions with a high-degree of conservation, reaching $77 \%$ of identity in the bZIP domain.

Consensus phylograms were constructed for $\mathrm{Clk}$ and vri orthologous genes, using a Bayesian inference with the BEAST v1.8 package [18] and the Maximumlikelihood (ML) approach designed in PHYML v3.0 [19]. All analyses were run in the Cipres Science Gateway environment [20]. Briefly, a prior tree was randomly generated in both types of phylogenetic reconstructions. The
Yule process of speciation was imposed for Bayesian trees. In this case, three independent runs were performed for $4 \times 10^{7}$ generations, with a burn-in of $10 \%$. Proper mixing of chains and convergence of parameters were confirmed by calculating the effective sample size (ESS) in Tracer v1.6 [21]. All considered parameters had ESS $>2 \times 10^{4}$. Maximum credibility tree was constructed with 12,000 trees (burn-in $=1,200$ ). Statistical support for clades was assessed by the posterior probability method and 1,000 bootstrap replicates in Bayesian and ML trees, respectively. ProtTest v3.0 [22] was used to elect the joint transform-domain-translated with unequal frequencies and four-gamma parameters $(J T T+\Gamma+\mathrm{I})$ was the best-fit model of amino acid substitution (Akaike Information Criterion corrected for number of samples and number of amino acids of each sequence) for $C l k$ and vri phylogenetic reconstructions. The Poisson model available in MEGA 5 [23] was used to estimate a species distance matrix and a heatmap was constructed in the $R$ environment [24].

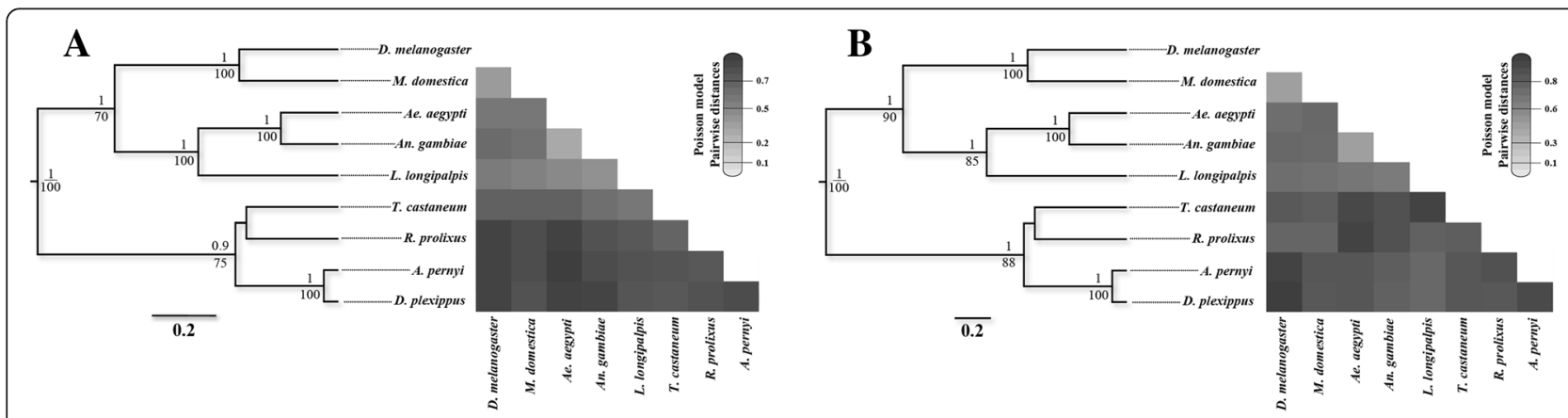

Fig. 2 Consensus phylograms based on the Bayesian inference Maximum-likelihood (ML) approach for Clk (a) and vri (b) genes and heatmaps illustrating pairwise genetic distance values. Posterior probabilities $>0.9$ and bootstrap values $>70$ are shown for each node (above and below each node, respectively). Darker colors of squares in the heatmaps represent larger distances (see scale bar) 
Phylogenetic analyses corroborated the previously known neopteran insect systematics (Fig. 2). The Nematocera suborder was recovered in a wellsupported cluster $(\mathrm{PP}=1$; bootstrap $=85 / 100)$ containing Clk and vri orthologues of L. longipalpis and of Ae. aegypti and An. gambiae (overall pairwise distance $=30 \%$ and $57 \%$ for Clk and vri, respectively). These sequences were closer to each other than to the other dipterans $D$. melanogaster and $M$. domestica (infraorder Muscomorpha; overall distance $=53-57 \%$ and $74-77 \%$ for Clk and vri, respectively). All dipteran sequences were well separated from others belonging to Coleoptera and Hemiptera (T. castaneum and $R$. prolixus, respectively, which clustered together), and to Lepidoptera orders (A. pernyi and $D$. plexippus), with distance values above $50 \%$ for $C l k$ and $80 \%$ for vri ortolog sequences.

We believe that the identification, annotation and phylogenetic analyses of $C l k$ and vri orthologues in $L$. longipalpis contribute with novel and important data towards a better understanding of this species' biology and its molecular clockwork. The coding sequences of the two clock genes provide useful information for probe design and open new possibilities for circadian expression and functional assays, as well as population genetic studies. Clock genes are suitable markers for identifying sibling species, since they are involved in species-specific behaviors that may lead to reproductive isolation and ultimately result in speciation [25-27].

A list of future prospects includes the silencing of $\mathrm{Clk}$ and vri expression (through RNAi) and analysis of the physiological and behavioral output of this manipulation. Also, the identification of conserved regions across the genes and the correct assignment of exon-exon boundaries are valuable information to design Exon-Priming Intron Crossing (EPICs) for molecular systematics studies in sand flies.

Finally, our findings also revealed a great divergence in CLK C-terminal sequence, with variation in the poly-Q position and length. In order to further investigate the functional significance of this region in sand flies, where it is much reduced in size, CLK chimeras can be constructed and tested in cell reporter assays. This will allow proper evaluation of its capacity to bind promoter elements and activate gene expression. Similarly, CLK chimeras in transgenic Drosophila lines may help to assess the ability of rescuing $C l k$-null mutant phenotypes. Curiously, a transcriptional activation domain very common in vertebrates (a.k.a. BCTR) is found in L. longipalpis cyc [10]. Assuming that CLK and CYC dimerize like in Drosophila, the BCTR could function to compensate the absence of CLK's poly-Q and provide an alternative model for clock genes regulation in the sand fly clockwork.

\section{Additional files}

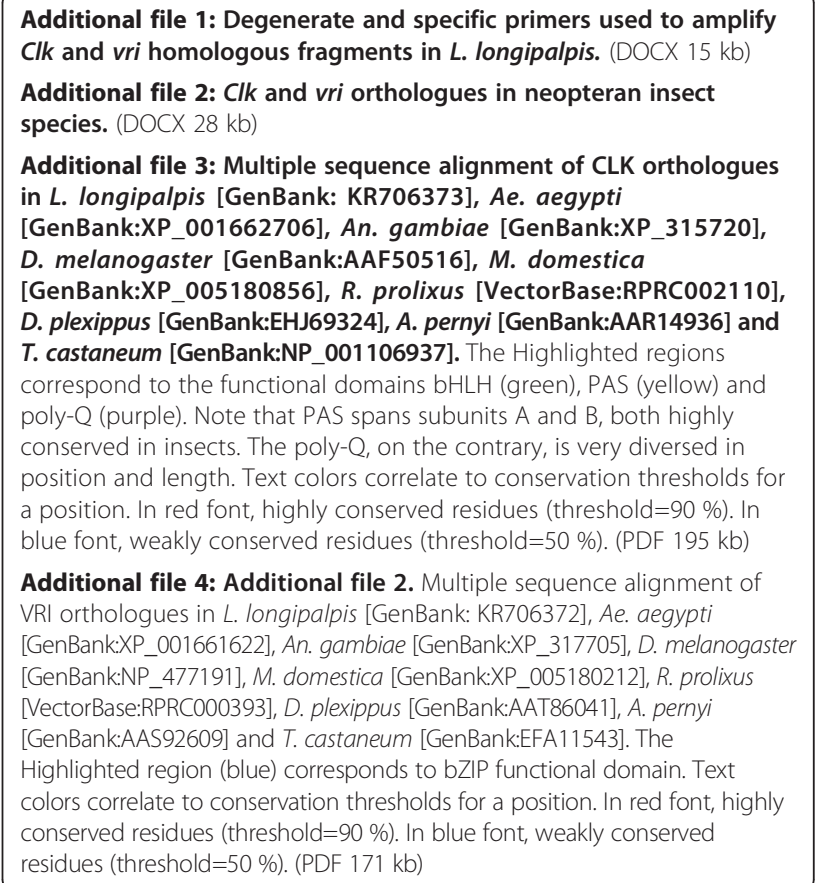

Competing interests

The authors declare that they have no competing interests.

\section{Authors' contributions}

JSMG carried out Clk cloning and drafted the manuscript. GBSR performed vri cloning. MGP did the phylogenetic analysis and wrote the manuscript. ACAMF and PRA helped with the cloning steps and sequencing. NAS was responsible for the acquisition of sand fly samples. RVB provided critical analysis of the data and helped to prepare and submit the manuscript. AAP coordinated the work, conceived the experimental design and supervised JSM and GBSR. All authors read and approved the final manuscript.

\section{Authors' information}

Alexandre Afranio Peixoto in memoriam.

\section{Acknowledgements}

We would like to thank Robson Costa da Silva for expert technical assistance. We also thank the PDTIS-FIOCRUZ DNA sequencing core for running sequencing reactions and Dr. Luciana Araripe for critical reading of the manuscript. This work was funded by the Howard Hughes Medical Institute and CNPq. We dedicate this paper to the memory of Dr. Alexandre Afranio Peixoto, an outstanding friend and scientist.

\section{Author details}

${ }^{1}$ Laboratório de Biologia Molecular, Instituto Oswaldo Cruz, Fundação Oswaldo Cruz (FIOCRUZ), Rio de Janeiro, Brazil. ²Mosquitos Vetores: Endossimbiontes e Interação Patógeno Vetor, Centro de Pesquisas René Rachou-Fiocruz, Belo Horizonte, Minas Gerais, Brazil. ${ }^{3}$ Laboratório de Epidemiologia e Sistemática Molecular, Instituto Oswaldo Cruz, Fundação Oswaldo Cruz (FIOCRUZ), Rio de Janeiro, Brazil. ${ }^{4}$ Laboratory of Systems Biology and Genetics, Institute of Bioengineering, School of Life Sciences, École Polytechnique Fédérale de Lausanne, Lausanne, Switzerland. 'Laboratório de Transmissores de Leishmanioses, Instituto Oswaldo Cruz, Fundação Oswaldo Cruz (FIOCRUZ), Rio de Janeiro, Brazil. ${ }^{6}$ Instituto Nacional de Ciência e Tecnologia em Entomologia Molecular (INCT-EM)/ CNPq, Rio de Janeiro, Brazil.

Received: 14 May 2015 Accepted: 28 September 2015

Published online: 06 October 2015 


\section{References}

1. Saunders DS, Steel CGH, Vafopoulou X, Lewis RD. Insect clocks. 3rd ed. Amsterdam: Elsevier; 2002.

2. Clements AN. The biology of mosquitoes. Vol. 2. Sensory reception and behaviour. New York: CABI Publishing; 1999.

3. Quinnell RJ, Dye C. An experimental study of the peridomestic distribution of Lutzomyia longipalpis (Diptera, Psychodidae). Bull Entomol Res. 1994;84:379-82.

4. Morrison AC, Ferro C, Pardo R, Torres M, Wilson ML, Tesh RB. Nocturnal activity patterns of Lutzomyia longipalpis (Diptera: Psychodidae) at an endemic focus of visceral leishmaniasis in Colombia. J Med Entomol. 1995;32:605-17.

5. Rivas GBS, Souza NA, Peixoto AA. Analysis of the activity patterns of two sympatric sandfly siblings of the Lutzomyia longipalpis species complex from Brazil. Med Vet Entomol. 2008:22:288-90.

6. Rivas GBS, Souza NA, Peixoto AA, Bruno RV. Effects of temperature and photoperiod on daily activity rhythms of Lutzomyia longipalpis (Diptera: psychodidae). Parasit Vectors. 2014;7:278.

7. Hardin PE. Molecular genetic analysis of circadian timekeeping in Drosophila. Adv Genet. 2011;74:141-73.

8. Ozkaya O, Rosato E. The circadian clock of the fly: a neurogenetics journey through time. Adv Genet. 2012;77:79-123.

9. Meireles-Filho ACA, Rivas GBS, Gesto JSM, Machado RC, Britto C, Souza NA et al. The biological clock of a hematophagous insect: locomotor activity rhythms, circadian expression and downregulation after a blood meal. Febs Letters. 2006;580:2-8

10. Meireles-Filho ACA, Amoretty PR, Souza NA, Kyriacou CP, Peixoto AA Rhythmic expression of the cycle gene in a hematophagous insect vector. BMC Mol Biol. 2006;7:38.

11. Gentile C, Meireles-Filho ACA, Britto C, Lima JBP, Valle D, Peixoto AA. Cloning and daily expression of the timeless gene in Aedes aegypt (Diptera: Culicidae). Insect Biochem Molec Biol. 2006;36:878-84.

12. Staden R, Judge DP, Bonfield JK. Analysing sequences using the staden package and EMBOSS. Introduction to bioinformatics. A theoretical and practical approach. Totawa: Humana Press Inc; 2003.

13. Allada R, White NE, So WV, Hall JC, Rosbash M. A mutant Drosophila homolog of mammalian Clock disrupts circadian rhythms and transcription of period and timeless. Cell. 1998;93:791-804.

14. Cyran SA, Buchsbaum AM, Reddy KL, Lin MC, Glossop NR, Hardin PE, et al. vrille, Pdp1, and dClock form a second feedback loop in the Drosophila circadian clock. Cell. 2003;112:329-41.

15. Finn RD, Bateman A, Clements J, Coggill P, Eberhardt RY, Eddy SR, et al. Pfam: the protein families database. Nucleic Acids Res. 2014;42(Database issue):D222-30.

16. Corpet F. Multiple sequence alignment with hierarchical clustering. Nucleic Acids Res. 1988;16:10881-90.

17. Henikoff S, Henikoff JG. Amino acid substitution matrices from protein blocks. Proc Natl Acad Sci U S A. 1992;89:10915-9.

18. Drummond AJ, Suchard MA, Xie D, Rambaut A. Bayesian phylogenetics with BEAUti and the BEAST 1.7. Mol Biol Evol. 2012;29:1969-73.

19. Guindon S, Dufayard JF, Lefort V, Anisimova M, Hordijk W, Gascuel O. New algorithms and methods to estimate maximum-likelihood phylogenies: assessing the performance of PhyML 3.0. Syst Biol. 2010;59:307-21.

20. Miller MA, Pfeiffer W, Schwartz T. Creating the CIPRES Science Gateway for inference of large phylogenetic trees. In: Proceedings of the Gateway Computing Environments Workshop (GCE), 14 November 2010; 1-8. IEEE. doi:10.1109/GCE.2010.5676129

21. Rambaut A, Suchard MA, Xie D, Drummond AJ. Tracer v1.6. 2014; Available from http://beast.bio.ed.ac.uk/Tracer

22. Darriba D, Taboada GL, Doallo R, Posada D. ProtTest 3: fast selection of best-fit models of protein evolution. Bioinformatics. 2011;27:1164-5.

23. Tamura K, Peterson D, Peterson N, Stecher G, Nei M, Kumar S. MEGA5: molecular evolutionary genetics analysis using maximum likelihood, evolutionary distance, and maximum parsimony methods. Mol Biol Evol. 2011;28:2731-9.

24. R Core Development Team. R: A language and environment for statistical computing. Vienna, Austria: R Foundation for Statistical Computing; 2010. Retrieved from http://www.R-project.org.

25. Coyne JA. Genetics and speciation. Nature. 1992;355:511-5.

26. Araki AS, Vigoder FM, Bauzer LGS, Ferreira GEM, Souza NA, Araújo IB, et al. Molecular and behavioural differentiation among Brazilian populations of
Lutzomyia longipalpis (Diptera: Psychodidae: Phlebotominae). PLoS Negl Trop Dis. 2009;3, e365.

27. Araki AS, Ferreira GE, Mazzoni CJ, Souza NA, Machado RC, Bruno RV, et al Multilocus analysis of divergence and introgression in sympatric and allopatric sibling species of the Lutzomyia longipalpis complex in Brazil. PLoS Negl Trop Dis. 2013;7, e2495.

\section{Submit your next manuscript to BioMed Central and take full advantage of:}

- Convenient online submission

- Thorough peer review

- No space constraints or color figure charges

- Immediate publication on acceptance

- Inclusion in PubMed, CAS, Scopus and Google Scholar

- Research which is freely available for redistribution 